\title{
Integrated Gabor Filter and Trilateral Filter for Exudate Extraction in Fundus Images
}

\author{
Kanika Bajaj \\ Department of Computer Science \& Engineering, Global Institute of Management and Emerging Technology \\ Amritsar, 143001, India \\ Email: bajajkanika07@gmail.com \\ Navjot Kaur \\ Department of Computer Science \& Engineering, Global Institute of Management and Emerging Technology \\ Amritsar, 143001, India \\ Email: navjot.632@gmail.com
}

\begin{abstract}
Image segmentation is the process of dividing an electronic digital image into numerous sub-images. Its objective is to categorize image into various regions in such a way that every potential object in image gets individual sector. Instinctive recognition of diabetic retinopathy wounds, like exudates can provide opportunity to identify certain diseases. Lack of accuracy in these techniques can lead to fatal results because of incorrect treatment. So, there is a great need for automation techniques with high accuracy for retinal disease identification. Several automation techniques have been proposed for retinal image analysis which can detect the exudates in fundus images in more promising manner. The related work has found that the issue of noise in fundus images is ignored in the majority of existing literature. Although Gabor filter bank has shown significant results over available techniques, but it is poor in its speed. Also it is not very efficient for multiple kinds of noises at a same time. Therefore to improve the accuracy of exudate extraction further a Hybrid Gabor filter bank with trilateral based filtering technique is proposed. This filtering will use improved trilateral filtering which enables us to detect exudates even in highly corrupted noisy images.
\end{abstract}

Index Terms - Image segmentation, Exudates segmentation, Hybrid Gabor filter, Fundus images

\section{INTRODUCTION}

Digital Image Processing is a method which deals with manipulation of a digital image with the help of a digital computer. DIP deals with the computer-based processing of digital images.

Image segmentation is the method of extracting required components from an image. Segmentation attempts to find an object of interest in the image. It includes dividing the image into 2 kinds of regions either object or background to extract the desired information. Segmentation takes place naturally in the human visual system. Humans are specialists in identifying patterns and shapes and making decisions primarily depending on the visual characteristics.

\section{A. Medical Image Segmentation}

Segmentation is very important in computer-based diagnosing of medical images. The target of image segmentation is to divide a picture in non-overlapping components on the basis of intensity and texture of the image. In segmentation technique, the primary step is denoising or image smoothing, to remove noise from the image like salt and pepper noise. Then, Thresholding is done, in which objects are extracted from the background based on the threshold values. This technique is helpful in segmenting images having light objects against a dark background or vice versa. Clustering technique is employed for identification of affected area in any medical image.

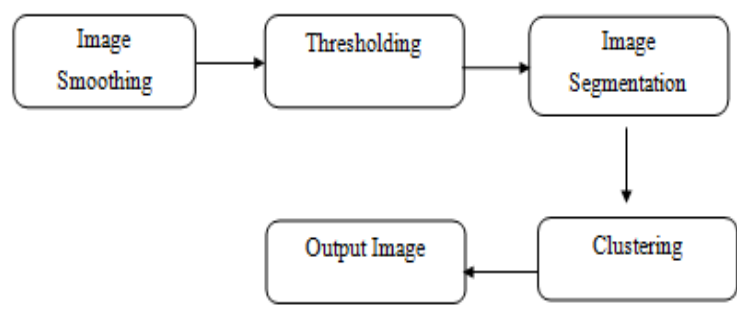

Fig.1. Basic steps of medical image segmentation

\section{B. Retinal Vessel Segmentation}

Basically, Retina is a layer of tissue at the inner surface of the eye which converts incoming light to the neural signal which is further processed by the brain. The appearance of the retina is a key indication of a wide range of eye diseases amongst individuals and could be diagnostic tool of a variety of problems concerning health and wellbeing of individuals. Many morphological properties of retinal arteries and veins (e.g. diameter, length, and branching angle) are of diagnostic significance and so are used in detecting and monitoring the disease progression, treating and evaluating some cardiovascular and ophthalmologic diseases. 
Characteristics of retinal images like the displacement of arteries and vessels in the OD (optic disk) are useful in automatic detection of various problems related to eye.

In this paper, an Integrated Gabor filter with trilateral based filtering is proposed for exudate extraction in fundus images. Section II describes various vessel segmentation techniques. The related work is discussed in Section III. Gaps in literature and proposed methodology are discussed in Section IV and V respectively. The performance analysis and results of the proposed technique are described in Section VI. Section VII discusses Conclusions.

\section{VESSEL SEGMENTTION TECHNIQUES}

Retinal vessel segmentation techniques describe various approaches to extract and analyze blood vessels from the fundus images. Numerous techniques for segmenting retinal vessels have been proposed over the time. Various techniques of vessel segmentation are-

\section{A. Multiscale Feature Extraction and Region Growing}

In this technique, parallelism is applied to the images by dividing an image into constituent sub images and every sub image can be processed simultaneously with other images within the available memory per processor to speed up the segmentation process. Region growing segmentation techniques work by starting from a seed point and combining the pixels depending on some prefixed criterion like similar intensity values and the pixels which lie spatially closer to the growing pixel. It is considered that the pixels having similar intensity values and nearby one another belong to the same object.

\section{B. Hybrid Filtering}

Hybrid filtering technique for segmenting retinal vessels is generally a pre-processing step in various areas to extract the vessels from the retinal images. In this technique, a Hessian matrix is used and filter is used. Orientation of the retinal vessels is provided by eigen vectors of the Hessian matrix and the edge constraints can be used to suppress the response of spurious boundary edges.

\section{Gabor Filter}

Dennis Gabor proposed the Gabor filtering technique which was later on used by Daugman. Gabor filter is a linear filtering technique which is widely used for edge detection. Gabor filters have an advantage of having frequency and orientation representations identical to the human visual system, and so it is very helpful in segmenting retinal vessel structures. Gabor filters are basically bandpass filters and are mainly used in feature extraction and texture analysis for processing images.

This filtering technique makes use of multi-scale Gabor filter characterized by one free parameter that determines the scale to identify the pixels called keypoints using the scale invariant feature transform (SIFT). Filter-response vectors derived from keypoints are used to initialize a clustering algorithm which generates textons which are eventually used for the classification of vessel pixels or non-vessel pixels in the input images. Then a process called model selection is used to identify keypoints from a small training set $(\mathrm{N}=$ 10) of retinal images. Then using these keypoints, a texton dictionary is built. In this second training stage, a $\mathrm{k}-\mathrm{NN}$ clustering algorithm is initialized with seeds found by matching keypoints identified by the model selection stage to those in the training set.

Leung and Malik characterize textons by its response to a filter bank $\left(\mathrm{F}_{1}, \mathrm{~F}_{2}, \ldots, \mathrm{F}_{\mathrm{n}}\right)$.

$$
\mathrm{R}=\mathrm{F}_{1} \times \mathrm{I}(\mathrm{x}, \mathrm{y}), \mathrm{F}_{2} \times \mathrm{I}(\mathrm{x}, \mathrm{y}), \ldots, \mathrm{F}_{\mathrm{n}}
$$

If the filter bank convolved with an input image I(x,y) the response at each pixel will depend on specific local structures and on the design of the filter bank.

\section{RELATED WORK}

This section describes the study of the related work done in the existing literature corresponding to retinal vessel segmentation.

Zhang et al. [2015] [6] presented an algorithm for segmenting retinal vessel structures which makes use of a texton dictionary to categorize vessel pixels or non-vessel pixels. This algorithm also uses Key points which are a small set of image features to derive the parameters for filtering. A Gabor filter bank presented in this paper has main aim of extracting key points from an image which represent significant information regarding vessel features with the help of SIFT algorithm.

Bhuiyan et al. [2013] [7] have discussed that the Agerelated macular degeneration (AMD) is the main reasons for vision defect among the aged people and identification of persons with first stages of AMD is vital while considering the preventative strategies for late AMD. They proposed a technique for drusen detection from standard color retinal images which enables quick, accurate and automated method to determine characteristics of drusen. The methodology achieved 100\% accuracy to detect drusen region.

Kamboj et al. [2013] [8] explained that enhancing a corrupted image by removing noise is an important step in digital image processing for better perception. Various filters are used for removing various types of the noise from the images. A Filter can be classified as linear filter (whose outcome is a linear function of its input) and nonlinear filter (whose outcome is a non-linear function of its input).

Esakkirajan et al. [2011] [9] presented a filter named Modified Decision Based Unsymmetrical Trimmed Median Filter (MDBUTMF). This technique is superior to existing algorithms for removing noise. The MDBUTMF algorithm gives is more efficient in comparison to existing techniques even in the presence of very high noise.

Giachetti et al. [2011] [10] gave a method to the segment the optic disk (OD) from the binary 
fundus images using morphological segmentation to decouple the vessel and background information. After this, a multi resolution optimization scheme is applied to the image to find elliptic contours.

Joshi et al. [2011] [11] presented an OD segmentation technique which combines the local information of images around every point of interest with the help of a multi-stage strategy.

Marin et al. [2011] [12] gave a methodology to isolate the blood vessels in the retinal images on the basis of a neural network (NN) scheme to classify pixels. In this technique, a 7-D vector composed of gray-level and moment invariants-based features is computed.

Santos et al. [2011] [13] proposed an efficient image processing method to segment drusen in the digital fundus images. The proposed algorithm aims at detecting AMD progression from a group of longitudinal images.

Fang et al. [2010] [14] gave a powerful technique to automatically segment hard exudates from the retinal image. This technique uses a course-to-fine strategy, in which initially a coarse value is obtained in which some negative samples are allowed, then these negative samples are get rid of step by step. To obtain a coarse segmentation result, they also gave a multi-scale background subtraction technique. HEs are extracted by a SVM classifier by excluding the optic disc region from the coarse value. The important points described in the paper are: (A) An efficient technique for segmentation of HE's (Hard Exudates); (B) A segmentation technique to mix multi-channel information; (C) Using the filter for segmenting the OD.

Gonzalez et al. [2010] [15] described a methodology for human retinal vessel segmentation with the help of graph cut methodology. In this method, firstly a graph is made by rough segmentation from a pre-processed image. The proposed technique was evaluated on two datasets and then compared with other techniques and it was concluded that this technique yields better performance than other unsupervised methods.

Hooshyar et al. [2010] [16] described a fuzzy clustering technique on the basis of Ant Colony Algorithm. Analysis of eigen values of Hessian matrix and Gabor filter bank are used to extract features of color retinal images. Artificial ants in the image make use of these features for finding and clustering processes. Lupascu et al. [2010] [17] presented a technique for vessel segmentation in which for every pixel in the image, a 41-D feature vector is made, which encodes the information on the local intensity structure, spatial properties, and geometry at multiple scales.

Welfer et al. [2010] [18] gave a technique to segment the OD (optic disk) in fundus images with the help of mathematical techniques. The experimental results are dependent upon two eye databases and prove that this technique can achieve a better performance in comparison to other algorithms.

Nayak et al. [2009] [19] has discussed that the Glaucoma is an illness of the optic nerve which primarily affects the optical disc (OD) because of enlargement of the cup size of the eye. Digital image process techniques, lik.e preprocessing, thresholding and morphological operations, are extensively used for the automated identification of optical disk and blood vessels.

Kong et al. [2008] [20] gave a color image segmentation scheme that combines the huepreservation color image equalization and segmentation techniques. The experimental results showed that the hemorrhagic spots and the important blood vessels are properly segmented as compared to the earlier technique.

\section{GAPS IN LITERATURE}

This section describes various issues in the existing technique. The literature survey has found that the majority of existing exudate segmentation techniques suffers from the following issues-

1. The issue of noise in fund us images is ignored in the majority of existing literature.

2. Although Gabor filter bank has shown significant results over available techniques, but it is poor in its speed.

3. The Gabor filter bank is rich in preserving the edges but not so efficient for high density of multiple noises.

\section{Methodology}

This section describes the sequence of steps followed in the proposed technique. Fig.2 is the flowchart showing steps for segmenting the retinal vessels from a human fundus image using the proposed methodology.

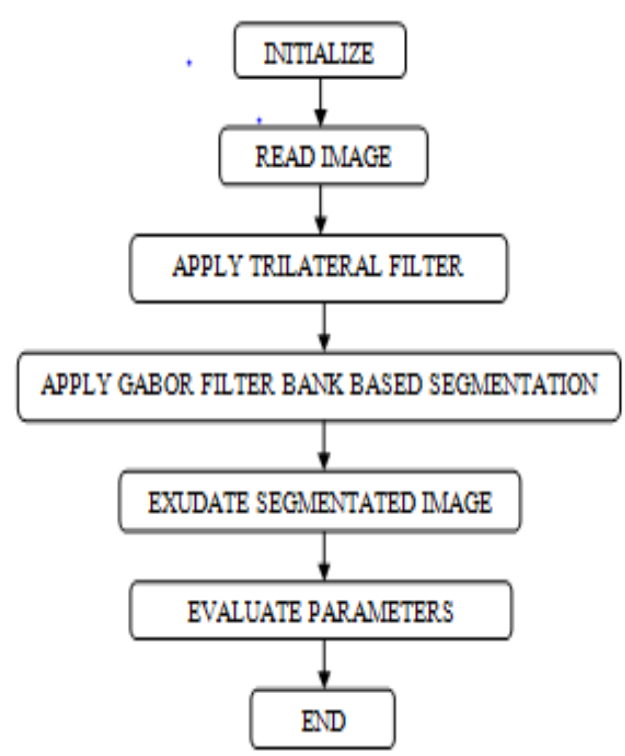

Fig.2. Flowchart of the proposed technique

The main steps of proposed technique are-

1. Input images in MATLAB. 
- Read the input retinal image.

- $\quad$ Read mask of the image

- Read the ground truth of an image.

2. Add salt and pepper noise to the image.

3. Apply trilateral filter to image for noise reduction.

4. Gray scaling of an image.

5. Apply Gabor filter bank based segmentation on gray scale image.

- Gabor filter bank is used at multiple scales to extract the features from the image.

- Then points of interest also known as key points are extracted from the retinal image.

- Textons are generated from the image. These textons are the small lines and blobs that are present in an image.

6. Retinal vessel Segmented image is obtained.

7. Evaluate parameters to compare existing and proposed techniques.

\section{PERformance AnAlysis AND RESUlts}

This section contains the cross validation considering existing and proposed techniques. The proposed algorithm using Hybrid Trilateral Gabor Filter Bank was applied on 10 images and their output was measured in terms of five parameters i.e. SN (Sensitivity), SP (Specificity), ACC (Accuracy), PPV (Positive and Negative predictive value), FDR (False Detection Rate).

In this section these parameters and their results are described by comparing these parameters w.r.t existing and proposed methodologies.

\section{1) SENSTIVITY $(S N)$}

Sensitivity is a statistical measure of the performance defining the quality or condition of being sensitive. Sensitivity can also be referred as true positive rate. It is the proportion of positives which can be identified accurately. Higher the value of sensitivity, better results can be obtained. It can be calculated as-

$$
S N=T P /(T P+F N)
$$

Table 1 shows the comparison of $\mathrm{SN}$ values for the existing and proposed algorithm. The value of proposed $\mathrm{SN}$ is higher as compared to existing $\mathrm{SN}$ for the 10 input images, thus it shows better result than the existing algorithm.
Table 1. The results of parameter SN for 10 input images.

\begin{tabular}{|c|c|c|}
\hline Image Name & Existing SN & $\begin{array}{c}\text { Proposed } \\
\text { SN }\end{array}$ \\
\hline Img1 & 0.8917 & 0.9974 \\
\hline Img2 & 0.9200 & 0.99707 \\
\hline Img3 & 0.9167 & 0.99922 \\
\hline Img4 & 0.8834 & 0.99995 \\
\hline Img5 & 0.9285 & 0.99979 \\
\hline Img6 & 0.9350 & 0.99952 \\
\hline Img7 & 0.8568 & 0.99976 \\
\hline Img8 & 0.9296 & 0.99754 \\
\hline Img9 & 0.8560 & 0.99991 \\
\hline Img10 & 0.8547 & 0.99984 \\
\hline
\end{tabular}

Fig.3 shows the graph of comparison of $\mathrm{SN}$ values for the Existing and proposed algorithm. The blue color in graph represents the SN value calculated using Gabor filter bank, maroon color represents the $\mathrm{SN}$ value calculated using hybrid trilateral Gabor filter. Value of $\mathrm{SN}$ is higher thus shows that proposed algorithms shows better result.

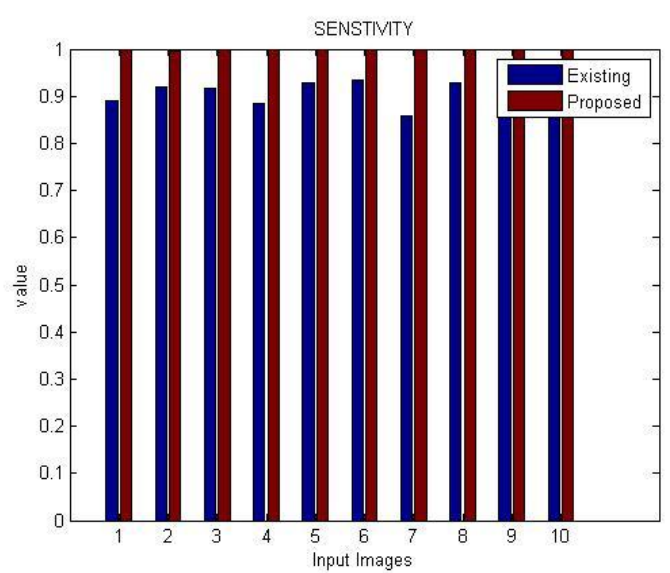

Fig.3. Comparative analysis of parameter SN for 10 input images

\section{2) $\operatorname{SPECIFICITY}(S P)$}

Specificity is a statistical measure of the performance defining the quality or condition of being specific. Specificity can also be referred as true negative rate. It is used to measure the proportion of negatives which can be correctly identified. Higher the value of specificity, better results can be obtained. It can be calculated as-

$$
S P=T N /(T N+F P)
$$

Table 2 shows the comparison of SP values for the existing and proposed algorithm. The value of proposed $\mathrm{SP}$ is higher as compared to existing $\mathrm{SN}$ for the 10 input images, thus it shows better result than the existing algorithm. 
Table 2. The results of parameter SP for 10 input images

\begin{tabular}{|c|c|c|}
\hline Image Name & Existing SP & $\begin{array}{c}\text { Proposed } \\
\text { SP }\end{array}$ \\
\hline Img1 & 0.9846 & 0.99136 \\
\hline Img2 & 0.9642 & 0.97606 \\
\hline Img3 & 0.9806 & 0.98094 \\
\hline Img4 & 0.9807 & 0.9853 \\
\hline Img5 & 0.9169 & 0.9258 \\
\hline Img6 & 0.9895 & 0.98713 \\
\hline Img7 & 0.9873 & 0.99152 \\
\hline Img8 & 0.9900 & 0.99551 \\
\hline Img9 & 0.9870 & 0.99875 \\
\hline Img10 & 0.9790 & 0.9786 \\
\hline
\end{tabular}

Fig.4 shows the graph of comparison of SP values for the existing and proposed algorithms. The blue color in graph represents the SP value calculated using Gabor filter bank, maroon color represents the SP value calculated using hybrid trilateral Gabor filter. Value of SP is higher thus shows that proposed algorithms shows better result.

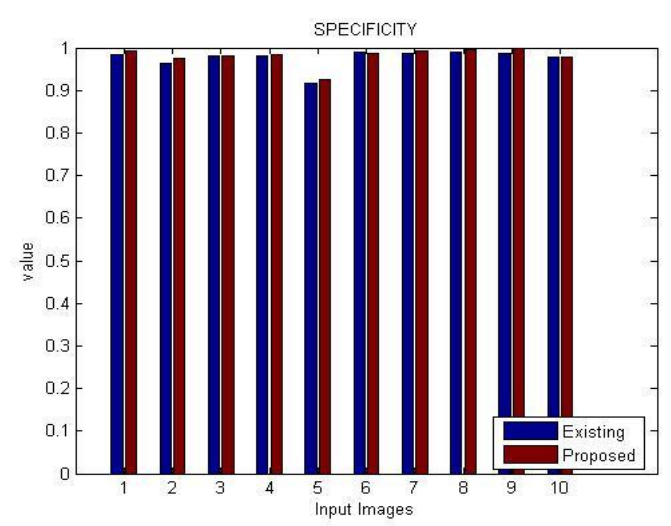

Fig.4. Comparative analysis of parameter SP for 10 input images

\section{3) ACCURACY (ACC)}

Accuracy is the amount of closeness of measurements of a quantity to that quantity's true value. Higher value of accuracy better will be the results. It can be calculated as-

$$
A C C=T P+T N /(T P+F P+F N+T N)
$$

Table 3 shows the comparison of ACC values for the existing and proposed algorithm. The value of proposed $\mathrm{ACC}$ is higher as compared to existing ACC for the 10 input images, thus it shows better result than the existing algorithm.
Table 3. The results of parameter ACC for 10 input images

\begin{tabular}{|c|c|c|}
\hline Image Name & $\begin{array}{l}\text { Existing } \\
\text { ACC }\end{array}$ & $\begin{array}{c}\text { Proposed } \\
\text { ACC }\end{array}$ \\
\hline Img1 & 0.9771 & 0.99977 \\
\hline Img2 & 0.9795 & 0.9997 \\
\hline Img3 & 0.9766 & 0.99993 \\
\hline Img4 & 0.9789 & 0.99999 \\
\hline Img5 & 0.9820 & 0.99998 \\
\hline Img6 & 0.9852 & 0.99995 \\
\hline Img7 & 0.9782 & 0.99998 \\
\hline Img8 & 0.9859 & 0.99983 \\
\hline Img9 & 0.9768 & 0.99999 \\
\hline Img10 & 0.9788 & 0.99998 \\
\hline
\end{tabular}

Fig.5 shows the graph of comparison of ACC values for the existing and proposed algorithms. The blue color in graph represents the ACC value calculated using Gabor filter bank, maroon color represents the ACC value calculated using hybrid trilateral Gabor filter. Value of ACC is higher thus shows that proposed algorithms shows better result.

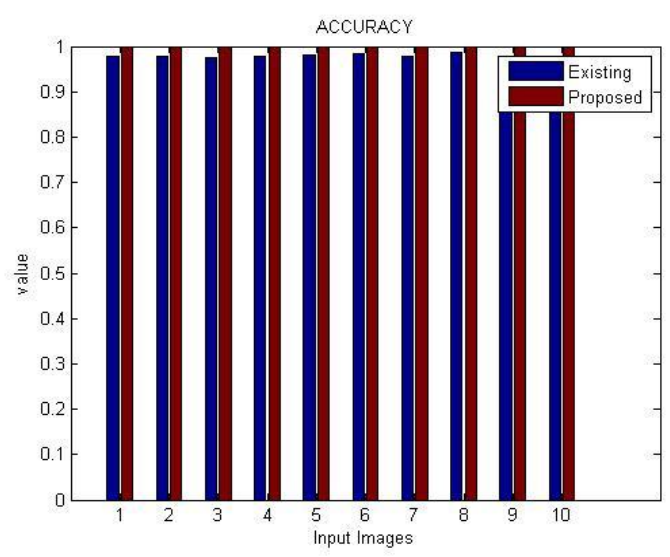

Fig.5. Comparative analysis of parameter SP for 10 input images

4) POSITIVE AND NEGATIVE PREDICTIVE VALUE
(PPV)

The PPV is defined as-

$$
P P V=T P /(T P+F P)
$$

Where TP is the event when the test makes a positive prediction, and the subject has a positive result. The FP is the event when the test makes a positive prediction and the subject has a negative result. 
Table 4 shows the comparison of PPV values for the existing and proposed algorithm. The value of proposed PPV is higher as compared to existing PPV for the 10 input images, thus it shows better result than the existing algorithm.

Table 4. The results parameter PPV for 10 input images.

\begin{tabular}{|c|c|c|}
\hline $\begin{array}{c}\text { Image } \\
\text { Name }\end{array}$ & Existing PPV & $\begin{array}{c}\text { Proposed } \\
\text { PPV }\end{array}$ \\
\hline Img1 & 0.8341 & 0.98319 \\
\hline Img2 & 0.8221 & 0.92277 \\
\hline Img3 & 0.7587 & 0.92732 \\
\hline Img4 & 0.8517 & 0.93251 \\
\hline Img5 & 0.8676 & 0.91704 \\
\hline Img6 & 0.8847 & 0.9016 \\
\hline Img7 & 0.8357 & 0.99504 \\
\hline Img8 & 0.8733 & 0.95597 \\
\hline Img9 & 0.8465 & 0.90771 \\
\hline Img10 & 0.8636 & 0.97322 \\
\hline
\end{tabular}

Fig.6 shows the graph of comparison of PPV values for the existing and proposed algorithms. In this graph, the SP values of existing images are compared with the values of proposed images. The blue color in graph represents the PPV value calculated using Gabor filter bank, maroon color represents the PPV value calculated using hybrid trilateral Gabor filter. Value of PPV is higher thus shows that proposed algorithms shows better result.

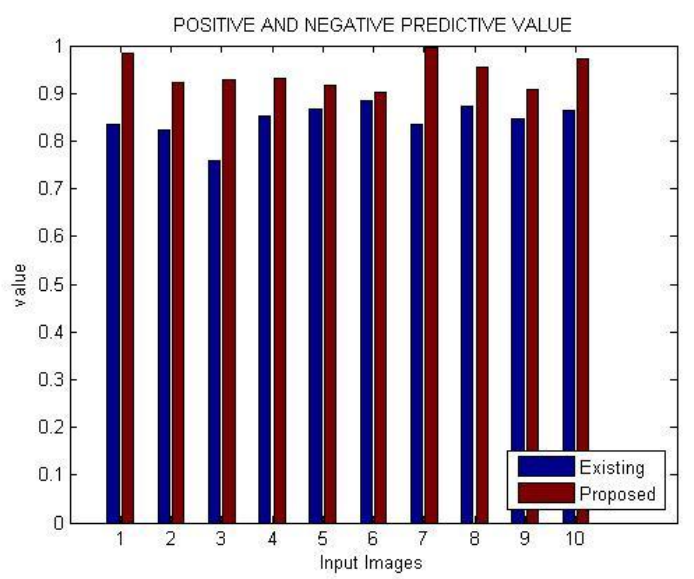

Fig.6. Comparative analysis of parameter PPV for 10 input images

\section{5) FALSE DETECTION RATE (FDR)}

The false discovery rate (FDR) is the expected proportion of false positives amongst the declared significant results.

Table 5 shows the comparison of FDR values for the existing and proposed algorithm. T, the value of proposed FDR is lower as compared to existing FDR for the 10 input images, thus it shows better result than the existing algorithm.
Table 5. The results of parameter FDR for 10 input images

\begin{tabular}{|c|l|l|}
\hline Image Name & $\begin{array}{l}\text { Existing } \\
\text { FDR }\end{array}$ & $\begin{array}{l}\text { Proposed } \\
\text { FDR }\end{array}$ \\
\hline Img1 & 0.1659 & 0.086951 \\
\hline Img2 & 0.1779 & 0.010055 \\
\hline Img3 & 0.2413 & 0.020098 \\
\hline Img4 & 0.1483 & 0.032047 \\
\hline Img5 & 0.1324 & 0.008978 \\
\hline Img6 & 0.1153 & 0.025696 \\
\hline Img7 & 0.1643 & 0.081974 \\
\hline Img8 & 0.1267 & 0.088262 \\
\hline Img9 & 0.1535 & 0.055885 \\
\hline Img10 & 0.1364 & 0.076124 \\
\hline
\end{tabular}

Fig.7 shows the graph of comparison of FDR values for the existing and proposed algorithms. In this graph, the FDR values of existing images are compared with the values of proposed images. The blue color in graph represents the FDR value calculated using Gabor filter bank, maroon color represents the FDR value calculated using hybrid trilateral Gabor filter. Value of FDR is lower thus shows that proposed algorithms shows better result.

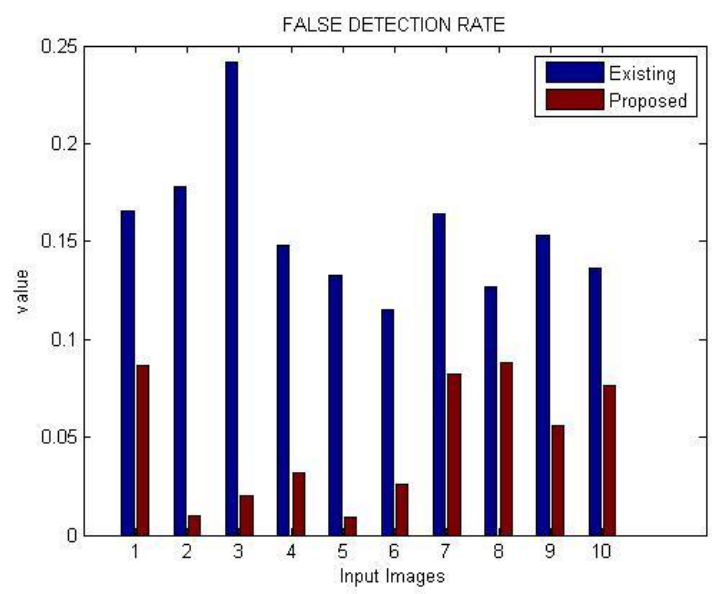

Fig.7. Comparative analysis of parameter FDR for 10 input images

\section{CONCLUSION}

Image segmentation technique is a method to partition an electronic digital image into numerous sub-images. Eye disorders are a common problem amongst many individuals. Identification of diabetic retinopathy lesions, such as exudates, in fundus images can help in early diagnosis of a problem. This technique shows better results however it is suffers from the effect of the noise. So a hybrid Gabor filter bank with trilateral based filtering technique is proposed in this dissertation to enhance the accuracy rate and to overcome the problem of noise from the images. The experimental results show that the Gabor filter bank based fundus segmentation has shown quite effective results while detecting the exudates in fundus images and enables us to detect exudates even 
in highly corrupted noisy images. This work has not considered the effect of low intensity in digital images. So in near future we will evaluate the effect of low intensity on digital images. Also some evolutionary optimization techniques will be used in future.

\section{REFERENCES}

[1] "Digital Image Processing",[ONLINE AVAILABLE]: http://www.tutorialspoint.com/dip/

[2] "Segmentation. In Introduction to Image Processing, Chapter 10", [ONLINE AVAILABLE]: http://www.cs.uu.nl/docs/vakken/ibv/reader/chapter10.pdf

[3] Orlando, J. I. and M. B. Blaschko "Learning FullyConnected CRFs for Blood Vessel Segmentation in Retinal Images" Medical Image Computing and Computer Assisted Intervention (MICCAI), 2014.

[4] Adithya Kusuma Whardana, Nanik Suciati,"A Simple Method for Optic Disk Segmentation from Retinal Fundus Image", IJIGSP, vol.6, no.11, pp.36-42, 2014.DOI: 10.5815/ijigsp.2014.11.05

[5] Krithikha Udayakumar, Paridhi P. Bharati, Shivani Verma, Malaya Kumar Nath,"Automatic Estimation of Vision Degradation from Color Fundus Image", IJIGSP, vol.7, no.11, pp.26-34, 2015.DOI: 10.5815/ijigsp.2015.11.04

[6] Zhang, Lei, Mark Fisher, and Wenjia Wang. "Retinal vessel segmentation using multi-scale textons derived from keypoints." Computerized Medical Imaging and Graphics, pp. 47-56, 2015

[7] Bhuiyan A, Karmakar C, Xiao D, Ramamohanarao K, Kanagasingam Y. "Drusen quantification for early identification of age related macular degeneration (AMD) using color fundus imaging" 35rd Annual international conference of the IEEE, engineering in medicine and biology society (EMBC). Osaka, Japan: IEEE; 2013. pp. 7392-7395, 2013.

[8] Priyanka Kamboj, Versha Rani,"Image Enhancement Using Hybrid Filtering Techniques ", International Journal of Science and Research, Vol 2, No. 6, June 2013.

[9] S. Esakkirajan, T. Veerakumar, Adabala N. Subramanyam, and C. H. PremChand,"Removalof High Density Salt and Pepper Noise Through Modified Decision Based Un symmetric Trimmed Median Filter", IEEE SIGNAL PROCESSING LETTERS, VOL. 18, NO. 5, MAY 2011.

[10] Giachetti, Andrea, Khai Sing Chin, Emanuele Trucco, Caroline Cobb, and Peter J. Wilson. "Multiresolution localization and segmentation of the optical disc in fundus images using inpainted background and vessel information." In Image Processing (ICIP), 2011 18th IEEE International Conference on, pp. 2145-2148. IEEE, 2011.

[11] Joshi, Gopal Datt, Jayanthi Sivaswamy, and S. R. Krishnadas. "Optic disk and cup segmentation from monocular color retinal images for glaucoma assessment." IEEE Transactions on Medical Imaging 30, no. 6 (2011): 1192-1205.

[12] Marín, Diego, Arturo Aquino, Manuel Emilio GegúndezArias, and José Manuel Bravo. "A new supervised method for blood vessel segmentation in retinal images by using gray-level and moment invariants-based features."IEEE transactions on medical imaging 30, no. 1 (2011): 146-158.

[13] Santos-Villalobos, Hector, Thomas Paul Karnowski, Deniz Aykac, Luca Giancardo, Yaquin Li, T. Nichols, K. W. Tobin, and Edward Chaum. "Statistical characterization and segmentation of drusen in fundus images." In Engineering in Medicine and Biology Society, EMBC, 2011 Annual International Conference of the IEEE, pp. 6236-6241. IEEE, 2011.
[14] Fang, Guoliang, Nan Yang, Huchuan Lu, and Kaisong Li. "Automatic segmentation of hard exudates in fundus images based on boosted soft segmentation." In Intelligent Control and Information Processing (ICICIP), 2010 International Conference on, pp. 633-638. IEEE, 2010.

[15] Salazar-Gonzalez, Ana G., Yongmin Li, and Xiaohui Liu. "Retinal blood vessel segmentation via graph cut." In Control Automation Robotics \& Vision (ICARCV), 2010 11th International Conference on, pp. 225-230. IEEE, 2010.

[16] Hooshyar, Sina, and Rasoul Khayati. "Retina vessel detection using fuzzy ant colony algorithm." In Computer and Robot Vision (CRV), 2010 Canadian Conference on, pp. 239-244. IEEE, 2010.

[17] Lupascu, Carmen Alina, Domenico Tegolo, and Emanuele Trucco. "FABC: retinal vessel segmentation using AdaBoost." IEEE Transactions on Information Technology in Biomedicine 14, no. 5 (2010): 1267-1274.

[18] Welfer, Daniel, Jacob Scharcanski, Cleyson M. Kitamura, Melissa M. Dal Pizzol, Laura WB Ludwig, and Diane Ruschel Marinho. "Segmentation of the optic disk in color eye fundus images using an adaptive morphological approach." Computers in Biology and Medicine 40, no. 2 (2010): 124-137.

[19] Nayak, Jagadish, Rajendra Acharya, P. Subbanna Bhat, Nakul Shetty, and Teik-Cheng Lim. "Automated diagnosis of glaucoma using digital fundus images." Journal of medical systems 33, no. 5 (2009): 337-346.

[20] Kong, Lingwang, Qiong Li, and Shanhu Huang. "Color Image Segmentation Scheme for Retinopathy Fundus." In Computer Science and Software Engineering, 2008 International Conference on, vol. 6, pp. 23

[21] Randy Cahya Wihandika,Nanik Suciati,"Retinal Blood Vessel Segmentation with Optic Disc Pixels Exclusion", IJIGSP, vol.5, no.7, pp.26-33, 2013.DOI: 10.5815/ijigsp.2013.07.04

\section{Authors' Profiles}

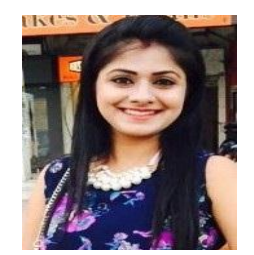

Kanika Bajaj, female, was born at Amritsar and completed bachelor degree in Computer Science and Engineering from Amritsar College of Engineering and Technology, Amritsar, India in 2011. She has done her master degree from Department of Computer Science \& Engineering, Global Institutes of Management and Emerging Technology, Amritsar, India in 2016.

She is currently working as an Assistant Professor in PG Department of CS and IT, D.A.V. College, Amritsar, India. Her research interests include digital image processing, pattern recognition and image segmentation.

Navjot Kaur, female, was born at Amritsar and completed master degree from Department of Computer Science \& Engineering, Global Institutes of Management and Emerging Technology, Amritsar, India in 2013. She is currently working as a faculty member at Department of Computer Science \& Engineering, Global Institutes of Management and Emerging Technology, Amritsar, India. Her research interests include digital image processing, computer vision and data mining. 
How to cite this paper: Kanika Bajaj, Navjot Kaur,"Integrated Gabor Filter and Trilateral Filter for Exudate Extraction in Fundus Images", International Journal of Image, Graphics and Signal Processing(IJIGSP), Vol.9, No.1, pp.10-17, 2017.DOI: $10.5815 /$ ijigsp.2017.01.02 